\title{
Long-Term Efficacy of Acute Thrombolytic Therapy for Myocardial Infarction
}

\author{
Haruo Hirayama, Akitada Ando*, Hirotaka Agetsuma**, Hideki Kato***, \\ Shoukoku LeE****, Hidehiko SAITO* and Iwao SotoBATA*****
}

We conducted a retrospective study (1981-1990) to determine whether the efficacy of intracoronary thrombolysis (ICT) could be evaluated from data obtained solely after recanalization. We investigated 55 successful ICT patients (38 with anterior and 17 with inferior myocardial infarction (MI)), and 31 control infarct patients without recanalization. The total serum creatine phosphokinase release ( $\Sigma \mathrm{CPK}$ ), the extent of infarction measured by Tl-201 single photon emission computed tomography (total DS) and the disturbance of regional wall motion (asyn.\%) were investigated as parameters for distinguishing the successful ICT and control groups. Discriminatory ability for the two groups was highest with the total DS in all patients. Only the total DS differed significantly between the two groups in patients with inferior infarction. Misidentification of control patients as successful patients was least frequent $\mathbf{2 5 . 5 \%}$ ) when using the total DS. These findings suggest that the effectiveness of ICT for acute MI may be assessed on the basis of data obtained solely after recanalization, with the total DS being particularly useful.

(Internal Medicine 33: 387-395, 1994)

Key words: intracoronary thrombolysis, $\Sigma$ CPK, Tl-201 SPECT, regional wall motion

\section{Introduction}

Recanalization therapy is effective for reducing the extent of myocardial necrosis in patients with acute myocardial infarction (MI). Intracoronary thrombolysis (ICT) $(1,2)$ and percutaneous transluminal coronary angioplasty (PTCA) (3) are now widely used alone and in combination for this purpose. The clinical use of tissue-type plasminogen activator (t-PA) has also begun. Intravenous thrombolytic therapy is being promoted as a primary care for MI (4). Should it prove feasible to administer emergency thrombolytic agents to Japanese patients with acute MI before transport to a cardiovascular center, it will probably be necessary to evaluate the efficacy of such treatment solely on the basis on laboratory data obtained after recanalization. The purposes of this study were 1) to determine whether parameters from data of creatine phosphokinase (CPK) release in the acute period, left ventriculogram and scintigram obtained 4-6 weeks after MI can evaluate the efficacy of thrombolytic therapy carried out during acute period of MI, and 2) to assess the most useful parameter among the three for evaluating the efficacy of thrombolytic therapy.

\section{Methods}

\section{Study population}

From a total 238 patients with acute MI admitted for emergency recanalization treatment between December 1981 and January 1990, we selected 55 patients who underwent successful ICT therapy (ICT group). They included 38 patients with anterior and 17 with inferior infarction, comprising 48 males and 7 females aged 42 to 79 years (mean: 60.9 years). Thirtyone MI patients, 28 males and 3 females aged 42 to 75 years (mean: 60.0 years) in whom ICT therapy resulted in failure acutely or chronically served as controls (control group), including 20 with anterior and 11 with interior infarction. The ICT group fulfilled the following criteria: severe chest pain persisting before ICT, successful ICT within a few hours of the onset of chest pain, and persistence of $R$ waves in the leads showing ST elevation.

The control group fulfilled the following criteria: failure to

\footnotetext{
From the Division of Cardiology, Cardiovascular Center, Suita, Nagoya Daini Red Cross Hospital, Nagoya, *the First Department of Internal Medicine, Nagoya University, Aichi, **Division of Cardiology, Toyota Memorial Hospital, Aichi, ***Internal Medicine, Kosai Kyouritsu Hospital, Shizuoka, ****Division of Cardiology, Hamamatsu Medical Center, Shizuoka and *****Faculty of Medicine, Fujita Health University, Aichi Received for publication March 4, 1993; Accepted for publication April 18, 1994 Reprint requests should be addressed to Dr. Haruo Hirayama, the Division of Cardiology, Cardiovascular Center, Nagoya Daini Red Cross Hospital, 2-9, Myoukencho, Showa-ku, Nagoya 466
} 


\section{HirayAma et al}

recanalization by ICT or reocclusion during the chronic phase of MI and absence of TIMI grade III or higher collaterals.

The other 183 patients were excluded from the study for the following reasons: emergency PTCA, cardiogenic shock (the blood pressure could not be maintained by intra-aortic balloon pumping or pressor agents), severe sequelae of cerebrovascular disease, surgery in the 10 days before MI, coagulation abnormalities, an age of 80 years or older, TIMI grade III (5) or higher collaterals on emergency coronary angiography, spontaneous recanalization, evidence of reinfarction, severe stenosis of coronary vessels other than the infarct-related artery, or death during the acute or chronic phase of MI. Table 1 shows the clinical characteristics of the patients in the ICT and control groups.

\section{Thrombolytic therapy}

Cardiac catheterization was carried out by the transfemoral route. After the vessels which were considered not responsible for infarction had been subjected to angiography, images of the culprit vessels were obtained from various directions. Nitro-

Table 1. Baseline Characteristics of the Subjects

\begin{tabular}{lccc}
\hline & \multicolumn{3}{c}{ Anteroseptal MI } \\
\hline $\begin{array}{l}\text { ICT group } \\
(\mathrm{n}=38)\end{array}$ & $\begin{array}{c}\text { Control group } \\
(\mathrm{n}=20)\end{array}$ & $\mathrm{p}$ value \\
\hline Age (range) & $58.3(42-73)$ & $57.9(42-75)$ & N.S. \\
Sex (M:F) & $33: 5$ & $19: 1$ & N.S. \\
$\begin{array}{l}\text { Occluded segments } \\
\text { (Seg 6:7) }\end{array}$ & $28: 10$ & $11: 9$ & N.S. \\
\hline
\end{tabular}

\begin{tabular}{lccc}
\hline & \multicolumn{3}{c}{ Inferior MI } \\
\hline $\begin{array}{l}\text { ICT group } \\
(\mathrm{n}=17)\end{array}$ & $\begin{array}{c}\text { Control group } \\
(\mathrm{n}=11)\end{array}$ & p value \\
\hline Age (range) & $66.7(56-79)$ & $63.7(47-72)$ & N.S. \\
Sex (M:F) & $15: 2$ & $9: 2$ & N.S. \\
$\begin{array}{l}\text { Occluded segments } \\
\begin{array}{l}\text { (Seg 1:2:3) } \\
\hline\end{array}\end{array}$ & $8: 7: 2$ & $4: 7: 0$ & N.S. \\
\hline
\end{tabular}

\begin{tabular}{lcc}
\hline & \multicolumn{2}{c}{ ICT group } \\
\hline & Anteroseptal MI & Inferior MI \\
\hline $\begin{array}{l}\text { Mean infarction } \\
\text { time (hours) }\end{array}$ & $3.6(1.8-8.8)$ & $3.9(1.7-8.9)$ \\
$\begin{array}{l}\text { MRS (\%) immediately } \\
\text { after reperfusion }\end{array}$ & $79.6(25-99)$ & $66.4(25-99)$ \\
$\begin{array}{l}\text { MRS (\%) in the } \\
\text { chronic period }\end{array}$ & $81.0(25-99)$ & $70.0(40-95)$ \\
\hline
\end{tabular}

ICT: intracoronary thrombolysis, MI: myocardial infarction, MRS: mean residual stenosis. glycerin $(0.5 \mathrm{mg})$ or isosorbide dinitrate $(5 \mathrm{mg})$ was infused into the culprit coronary artery to exclude infarction due to coronary spasm. Urokinase $(24,000$ units/min) was infused into the culprit artery over $10 \mathrm{~min}$ by mixing with autologous blood, and then angiography was repeated. This procedure was repeated until the thrombi had been completely lysed or until a total urokinase dose of 960,000 units was administered. Concomitant PTCA was not performed unless there was delayed peripheral runoff of contrast. After the administration of urokinase, heparin was administered at a dose of 12,000-20,000 units daily for several days, followed by oral anticoagulants until follow-up angiography was performed during the chronic period.

\section{Determination of $C P K$}

Serum CPK levels were determined every 4 hours from immediately after admission to the coronary care unit for the first 48 hours and then every 12 hours for 4 days (Fig. 1). The rate of disappearance of CPK from the blood was calculated for each patient from the changes in the serum CPK level over time and the descending phase of the disappearance curve. Then total CPK release ( $\Sigma \mathrm{CPK})$ was calculated according to the formulas of Shell et al (6), Roberts et al (7), and Norris et al (8) as follows:

$$
\begin{aligned}
\Sigma C P K= & \int_{0}^{t} f(t) d t(I U / m l) \text { (Norris et al) } \\
& \int_{0}^{t} f(t) d t: \text { total cardiac CPK release }(I U / m l) \\
\frac{d E(t)}{d t}= & f(t)-K_{D} E(t) d t \\
& \int_{0}^{t} f(t) d t=E(t)+K_{D} \int_{0}^{t} E(t) d t
\end{aligned}
$$

\section{Tl-201 SPECT}

SPECT (9) using Tl-201 (2 mCi) was performed during the chronic phase of MI (4-6 weeks after onset). The long axis of the left ventricle was divided into 6 equal segments and circumferential profile analysis (10) was carried out in each of the 6 short-axis views (Fig. 2). In the short-axis view, the 6 o'clock direction from the center of the heart was regarded as $0^{\circ}$, and the cross-sectional view of the heart was divided clockwise into 60 sectors of $6^{\circ}$ each. A percent thallium (\% Tl) uptake curve was prepared by setting the maximum profile count at $100 \%$. The mean -2 standard deviations (SD) of the values determined in normal individuals was defined as the lower limit of the normal range, and the area of the \% Tl uptake curve below this limit was regarded as indicating the defect size. The sum of the defects in the 6 short-axis views was defined as the total defect size (DS). SPECT was performed with a general-purpose collimator (GCA90A, Toshiba, Tokyo, Japan), and data processing was done with an ADAC System III.

\section{Quantitative evaluation of regional wall motion}

Regional wall motion was evaluated by left ventriculography 

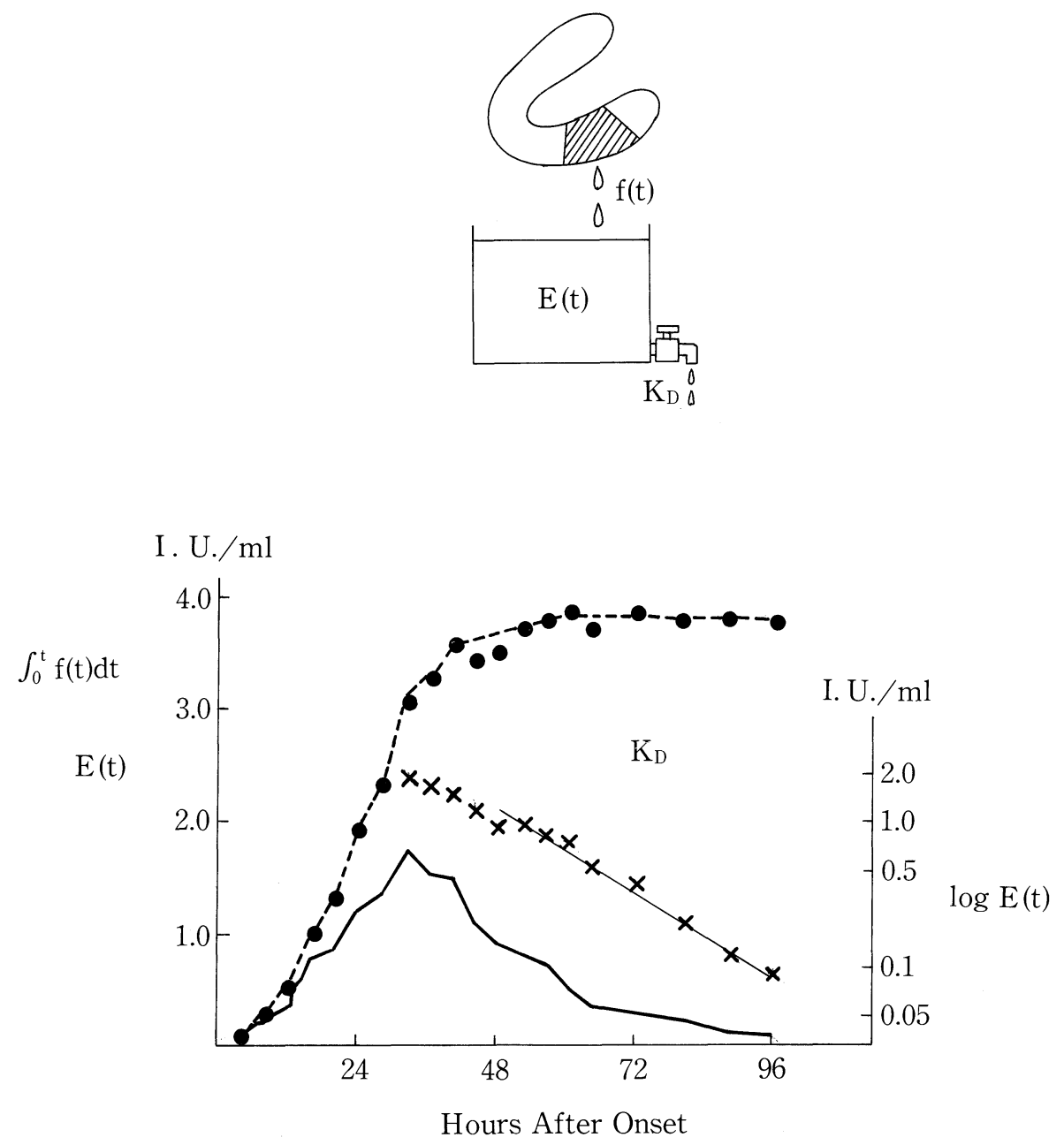

$f(t)$ : rate of change of CPK activity due to enzyme release from the heart (IU/ml.min) $\mathrm{E}(\mathrm{t})$ : blood CPK activity $(\mathrm{IU} / \mathrm{ml})$

$\mathrm{K}_{\mathrm{D}}$ : fractional rate of disappearance of $\mathrm{CPK}$ from the blood

- serial serum CPK activity levels

$X$ a semi-logarithmic replot of the same values from the peak activity downwards

- calculated integral values

Fig. 1. Measurement of the serum creatine phosphokinase (CPK) activity and calculation of total CPK release ( $\Sigma \mathrm{CPK})$.

in the $30^{\circ}$ right anterior oblique view using center-line method (11) 4 to 6 weeks after MI (Fig. 3). According to Sheehan's method, segments 10 to 66 (numbered clockwise from the aortic valve) were defined as the anterior wall region and segments 51 to 80 were regarded as the inferior wall region. Left ventricular wall motion below the mean $-1.5 \mathrm{SD}$ of the values in normal individuals was regarded as abnormal, and the percentage of segments showing abnormal motion was calculated as the percent asynergy (asyn.\%).

The percent stenosis of the culprit coronary artery was determined from actual measurements (diameter at the stenosis/ mean of the normal diameters proximal and distal to the stenosis), but it was defined as $99 \%$ when visualization of the vessel distal to the stenosis was delayed (12).

\section{Statistical analysis}

Statistical analysis was done by multivariate canonical discriminant analysis, using the patient group (ICT or control group) as the dependent variables and the $\Sigma \mathrm{CPK}$, total DS, and asyn.\% as independent variables. Stratified analyses were carried out using these parameters for patients in the anterior infarction subgroup and the inferior infarction subgroup. A p value less than 0.05 was accepted as indicating statistical significance. Parameters determined included the standard regression coefficient, which was used to assess the relative weighting of each parameter in categorizing the patients (the contribution increasing with an increase in the absolute value).

Differences in the mean values of $\Sigma C P K$, total DS, and asyn.\% between the ICT group and the control group were 


\section{HiRAYAMA et al}
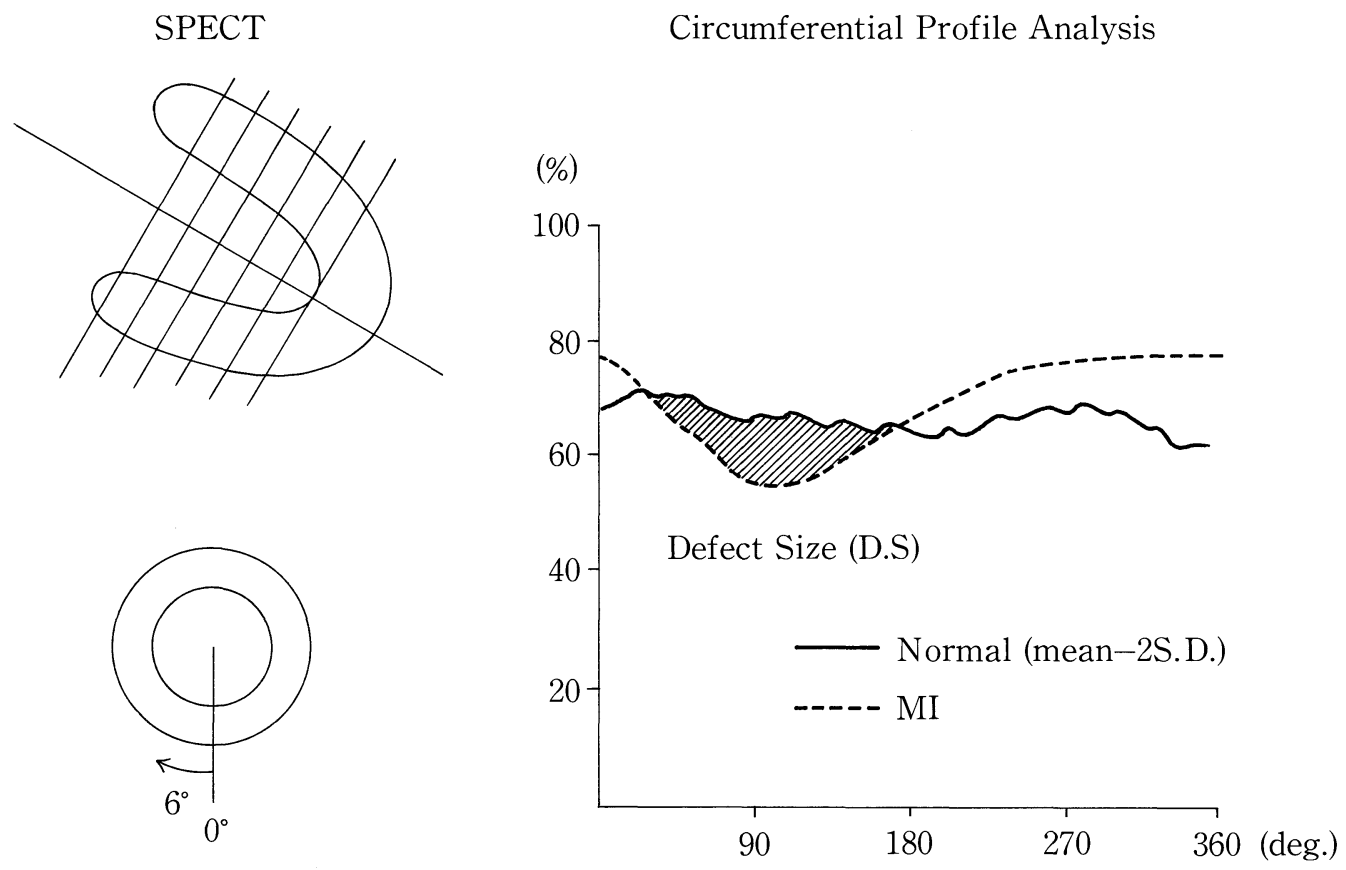

total DS. Sum of DS in 6 short-axis views

Fig. 2. Determination of the size of myocardial defect by Tl-201 SPECT. The long axis of the left ventricle (LV) was divided into 6 equal segments. In the short-axis view, the LV was divided clockwise into 60 sectors of $6^{\circ}$ from the $60^{\prime}$ clock position. The area of the percent thallium (\% $\mathrm{Tl}$ ) uptake curve below the mean -2 SD for normal individuals was regarded as the defect size (DS, \%deg.) and the sum of DS in the 6 short-axis views was defined as the total DS.

examined by one-way analysis of variance. The results given in parentheses in Tables $2-4$ are the frequencies of erroneous discrimination obtained by reciprocal examination.

\section{Results}

In the ICT patients with anterior infarction, the mean interval between the onset of infarction and recanalization (infarction time) was 3.6 hours (range: $1.8-8.8$ hours), the mean residual stenosis immediately after reperfusion was $79.6 \%$ (range: 25 $99 \%$ ), and the mean residual stenosis in the chronic period was $66.4 \%$ (range: $25-99 \%$ ). The corresponding figures for the ICT patients with inferior infarction were 3.9 hours (range: $1.7-8.9$ hours), $81.0 \%$ (range: $25-99 \%$ ), and $70.0 \%$ (range: $40-95 \%$ ).

Figure 4 shows a comparison of total DS, $\Sigma$ CPK and asyn.\% between ICT and control groups in subgroups of anterior and inferior MI. Significant differences between the ICT and the control group in the anterior infarction subgroup were observed for $\sum$ CPK $(\mathrm{p}<0.01)$, total DS $(\mathrm{p}<0.01)$, and asyn. \% $(\mathrm{p}<0.01)$. However, the only significant difference between the ICT and control groups was found in the total DS $(p<0.05)$ for the inferior infarction subgroup (one-way analysis of variance). There were no significant differences between the ICT and control groups in $\Sigma \mathrm{CPK}$ and asyn.\% in the inferior infarction subgroup (Fig. 4).

The frequency of erroneous discrimination obtained by reciprocal examination appeared to be lowest $(29.0 \%)$ by the asyn.\% in all patients compared with the frequency obtained by total DS (38.0\%) and $\Sigma$ CPK (44.2\%) (Table 2). The frequency of erroneous discrimination by the total DS in the anterior infarction subgroup appeared to be lowest $(25.5 \%)$ compared with those obtained by $\Sigma$ CPK $(34.5 \%)$ and asyn.\% (28.6\%) (Table 3). The frequencies of erroneous discrimination obtained by reciprocal examination were $35.8 \%$ by total DS and $35.6 \%$ by asyn. $\%$ in the inferior infarction subgroup whereas that by $\Sigma C P K$ was $38.8 \%$ (Table 4 ). Sensitivity was the highest with total DS compared with asyn.\% and $\Sigma \mathrm{CPK}$ in all patients, the anterior infarction and inferior infarction subgroups (Tables 2-4). Specificity was highest with asyn.\% compared with total DS and $\Sigma \mathrm{CPK}$ in all patients and inferior infarction subgroups (Tables $2-4$ ).

\section{Discussion}

In the present study, we found that, for identifying successful ICT patients, the total DS showed the lowest frequency of erroneous discrimination obtained by reciprocal examination in the anterior and inferior infarction subgroups compared with $\Sigma \mathrm{CPK}$ and asyn.\%, and that sensitivity and specificity were highest with total DS and asyn.\%, respectively. These findings suggested that assessment of the effect of recanalization therapy for acute MI can be based exclusively on data obtained in the 


\section{Efficacy of Intracoronary Thrombolysis}

Centerline Method
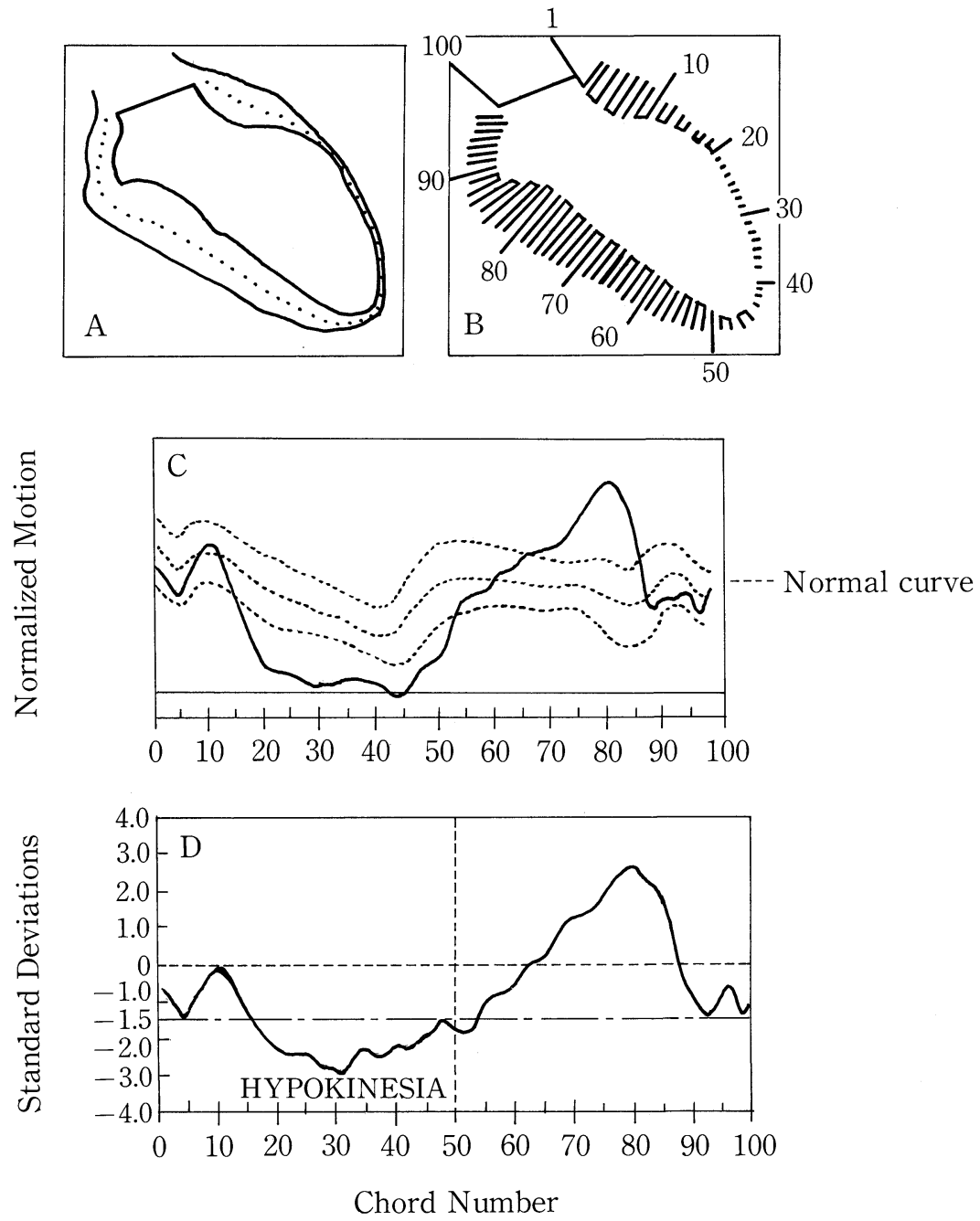

Chord No. 10-66; Anterior wall Chord No. 51-80; Inferior wall

Fig. 3. Quantitation of regional wall motion in the $30^{\circ}$ right anterior oblique view. The left ventricle was divided clockwise into 100 equal segments. Left ventricular wall motion below the mean $-1.5 \mathrm{SD}$ for normal individuals was regarded as abnormal, and the percentage of segments showing abnormal motion was defined as asyn.\% (=\% of asynergy).

chronic period, with estimation of total DS obtained by Tl-201 SPECT being particularly useful for this purpose.

We studied patients in whom the recanalization by thrombolytic therapy could be confirmed during the acute period of MI. We excluded patients who showed early spontaneous recanalization and those with TIMI grade III or higher collaterals in the acute or chronic period in order to eliminate the effect of collateral circulation. For comparison with this group undergoing successful early recanalization without reocclusion, patients in whom recanalization was not achieved or who developed reocclusion during follow up were selected as a control group from among those who had undergone recanalization therapy.
Various reports (6-8) have stated that the $\Sigma$ CPK value is closely correlated with the size of the myocardial defect observed pathologically and have suggested its use as a quantitative parameter for assessing infarction. While Uematsu and Hirayama reported that peak CPK and $\Sigma C P K$ values showed a strong correlation in both recanalized and non-recanalized patients, they did not recommend the use of peak CPK for comparing the two groups (13). We, therefore, used $\Sigma \mathrm{CPK}$ as a parameter of infarct size, with the total DS calculated from Tl201 SPECT images of myocardial blood flow as an additional parameter.

One-way analysis of variance showed significant differences in all three parameters between the ICT and control 
Table 2. Multivariate Analysis of All Subjects

\begin{tabular}{|c|c|c|c|}
\hline & $\Sigma \mathrm{CPK}$ & Total DS & asyn.\% \\
\hline $\begin{array}{l}\text { Standard regression } \\
\text { coefficient }\end{array}$ & -0.441 & 1.082 & 0.572 \\
\hline $\begin{array}{l}\mathrm{p} \text { value for a single } \\
\text { parameter }\end{array}$ & 0.0025 & 0.0001 & 0.0006 \\
\hline \multirow[t]{2}{*}{$\sum \mathrm{CPK}$} & \multicolumn{3}{|c|}{ Discrimination using $\Sigma \mathrm{CPK}$ alone (44.2\%) } \\
\hline & ICT group & Control group & Total \\
\hline ICT group & 33 & 22 & 55 \\
\hline Control group & 15 & 16 & 31 \\
\hline Total & 48 & 38 & 86 \\
\hline \multirow[t]{2}{*}{ Total DS } & \multicolumn{3}{|c|}{ Discrimination using total DS alone $(38.0 \%)$} \\
\hline & ICT group & Control group & Total \\
\hline ICT group & 38 & 17 & 55 \\
\hline Control group & 14 & 17 & 31 \\
\hline Total & 52 & 34 & 86 \\
\hline \multirow[t]{2}{*}{ asyn.\% } & \multicolumn{3}{|c|}{ Discrimination using asyn.\% alone (29.0\%) } \\
\hline & ICT group & Control group & Total \\
\hline ICT group & 32 & 23 & 55 \\
\hline Control group & 5 & 26 & 31 \\
\hline Total & 37 & 49 & 86 \\
\hline
\end{tabular}

asyn.\%: \% of asynergy of left ventricular wall motion, DS: defect size calculated from the myocardial perfusion image of thallium-201, ICT: intracoronary thrombolysis, $\Sigma \mathrm{CPK}$ : the total serum creatine phosphokinase release.

groups with anterior infarction, while only the total DS showed a significant difference in the inferior infarction subgroup. The frequency of erroneous discrimination obtained by reciprocal examination was the lowest when using the total DS in the anterior infarction subgroup, but the total DS and asyn.\% showed nearly the same frequency of erroneous discrimination in the inferior infarction subgroup.

The difficulty of quantitating inferior infarction has been noted by many investigators. Mahmarian et al (14) compared defect sizes determined by Tl-201 SPECT and by CK-MB values and found a high correlation between them in patients with anterior infarction $(\mathrm{r}=0.91)$, but a poor correlation in those with inferior infarction $(\mathrm{r}=0.50)$. They suggested that the correlation decreased in inferior infarction, which is often complicated by right ventricular infarction, because the former parameter reflects the size of the left ventricular defect, while the latter parameter reflects total myocardial necrosis (14). Thus, the difficulty in quantitating inferior infarction might be one of the reasons for lower discrimination between ICT and control
Table 3. Multivariate Analysis of the Anterior MI Subgroup

\begin{tabular}{lccc}
\hline & $\Sigma \mathrm{CPK}$ & Total DS & asyn.\% \\
\hline $\begin{array}{l}\text { Standard regression } \\
\text { coefficient }\end{array}$ & -0.242 & 1.160 & 0.287 \\
$\begin{array}{l}\text { p value for a single } \\
\text { parameter }\end{array}$ & 0.0013 & 0.0001 & 0.0023 \\
\hline
\end{tabular}

\begin{tabular}{lccc}
\hline$\Sigma C P K$ & \multicolumn{3}{c}{ Discrimination using $\Sigma$ CPK alone (34.5\%) } \\
\hline & ICT group & Control group & Total \\
\hline ICT group & 27 & 11 & 38 \\
Control group & 8 & 12 & 20 \\
\hline Total & 35 & 23 & 58 \\
\hline
\end{tabular}

\begin{tabular}{lccc}
\hline Total DS & \multicolumn{4}{c}{ Discrimination using total DS alone $(25.5 \%)$} \\
\hline & ICT group & Control group & Total \\
\hline ICT group & 30 & 8 & 38 \\
Control group & 6 & 14 & 20 \\
\hline Total & 36 & 22 & 58 \\
\hline \multicolumn{4}{c}{ Discrimination using asyn.\% alone $(28.6 \%)$} \\
\hline asyn.\% & ICT group & Control group & Total \\
\hline & 22 & 16 & 38 \\
\hline ICT group & 3 & 17 & 20 \\
\hline Tontrol group & 25 & 33 & 58 \\
\hline
\end{tabular}

Symbols are the same as in Table 2.

groups in the inferior infarction subgroup as compared with the anterior infarction subgroup.

While previous studies of ventricular wall motion have employed both the radial and rectangular methods (15-17), we used the center-line method because it allowed quantitative measurement of the area and the severity of abnormal wall motion. Although all three of the quantitative parameters we used have been studied previously (18), we found that total DS made the greatest contribution to discriminating ICT from control patients in both the anterior and inferior infarction subgroups. Rahimtoola (19) introduced the concept of stunned myocardium whereby the impairment of myocardial contractility remains long after severe ischemic attack even if the myocardium is viable. Braunwald and Rutherford (20) demonstrated the evidence of the hibernating myocardium, in that ischemia induces long-lasting ventricular dysfunction. In patients with stunned and hibernating myocardium (19-23), wall motion does not immediately recover after the myocardium is salvaged by recanalization following acute MI. Schofer and Mathy (24) 
Table 4. Multivariate Analysis of the Inferior MI Subgroup

\begin{tabular}{lccc}
\hline & $\Sigma \mathrm{CPK}$ & Total DS & asyn.\% \\
\hline $\begin{array}{l}\text { Standard regression } \\
\text { coefficient }\end{array}$ & -0.517 & 1.361 & 0.130 \\
$\begin{array}{l}\text { p value for a single } \\
\text { parameter }\end{array}$ & 0.0856 & 0.0156 & 0.2050 \\
\hline
\end{tabular}

\begin{tabular}{lccc}
\hline$\Sigma \mathrm{CPK}$ & \multicolumn{3}{c}{ Discrimination using } \\
& ICPK alone & $(38.8 \%)$ \\
\hline ICT group & Control group & Total \\
Control group & 10 & 7 & 17 \\
\hline Total & 4 & 7 & 11 \\
\hline
\end{tabular}

\begin{tabular}{lccc}
\hline Total DS & \multicolumn{3}{c}{ Discrimination using total DS alone (35.8\%) } \\
\hline & ICT group & Control group & Total \\
\hline ICT group & 11 & 6 & 17 \\
Control group & 4 & 7 & 11 \\
\hline Total & 15 & 13 & 28 \\
\hline
\end{tabular}

\begin{tabular}{lccc}
\hline asyn.\% & \multicolumn{3}{c}{ Discrimination using asyn.\% alone $(35.6 \%)$} \\
\hline & ICT group & Control group & Total \\
\hline ICT group & 8 & 9 & 17 \\
Control group & 2 & 9 & 11 \\
\hline Total & 10 & 18 & 28 \\
\hline
\end{tabular}

Symbols are the same as in Table 2.

have reported that neither left ventricular function nor regional wall motion was significantly correlated with the defect size estimated by Tl-201 SPECT in patients who underwent recanalization therapy for acute MI. They suggested that this poor correlation arose because regional wall motion is impaired at the margin of the infarct zone, despite the presence of surviving myocardium (stunned myocardium) (24). Stunned myocardium at the margin of the infarct zone probably influenced the present evaluation. The total DS allowed quantitation of the injured, but viable, myocardium that does not contribute to cardiac contraction at the time of scintigraphy and ventriculography 4-6 weeks after MI. This fact might explain in part the superiority of total DS for distinguishing the ICT group from the control group as compared with asyn.\% and $\Sigma \mathrm{CPK}$ in the present study.

The results of the reciprocal examination suggested that misidentification between the ICT and control groups was least likely to occur when using discrimination according to asyn.\% if left ventricular wall motion had been restored. However, left ventricular wall motion is very sensitive to ischemia, and the stunned myocardium and hibernation produced by coronary occlusion may persist for 4-6 weeks or more after recanalization (25). Therefore, there is a possibility of underestimating the effect of recanalization on acute $\mathrm{MI}$ if the outcome is evaluated on the basis of wall motion during this period.

\section{Limitations}

There are some limitations in the present study. First, we selected 55 patients for this study from 238 patients who underwent recanalization. However, the study population was small. Additional studies with a larger number of patients will be needed. Second, the control group of the present study included patients with reocclusion because we intended to distinguish exclusively the successful ICT patients at the chronic period from patients with unsuccessful ICT and reocclusion. It might provide clearer results to compare the successful ICT group with a control group consisting of patients with unsuccessful ICT. However, we think that the grouping used in this study may be more practical.

\section{Conclusion}

Our findings suggested that assessment of the effect of recanalization therapy for acute MI can be based exclusively on data obtained in the chronic period 4-6 weeks after the event, with estimation of total DS obtained by Tl-201 SPECT being particularly useful for this purpose.

Acknowledgments: The authors gratefully acknowledge assistance with the multivariate analysis from Mr. Yoichi Higashibeppu and Mr. Hideki Origasa, as well as that of Mr. Kunishige Sugiyama (head of the Second Division, Center of Clinical Pharmacology, Eisai Co., Ltd., Tokyo, Japan).

\section{References}

1) Rentrop KP, Blanke H, Karsch KR, et al. Acute myocardial infarction: Intracoronary application of nitroglycerin and streptokinase in combination with transmural recanalization. Clin Cardiol 2: 354, 1979.

2) Kanbara H. A study of efficacy of intracoronary thrombolysis therapy for acute myocardial infarction. Saishinigaku 39: 1059, 1984 (in Japanese).

3) Gruentig AR, Senning A, Siegenthaler WE. Nonoperative dilatation of coronary artery stenosis: Percutaneous transluminal coronary angioplasty. N Engl J Med 301: 61, 1979.

4) Sheehan FH. The effect of intravenous thrombolytic therapy on left ventricular function: A report on tissue-type plasminogen activator and streptokinase from the Thrombolysis in Myocardial Infarction (TIMI Phase I) Trial. Circulation 75: 817, 1987.

5) TIMI study group. The thrombolysis in myocardial infarction (TIMI) trial. N Engl J Med 312: 932, 1985.

6) Shell WE, Kjekshus JK, Sobel BE. Quantitative assessment of the extent of myocardial infarction in the conscious dog by means of analysis of serial changes in serum creatine phosphokinase activity. J Clin Invest 50: 2614, 1971.

7) Roberts R, Henry PD, Sobel BE. An improved basis for enzymatic estimation of infarct size. Circulation 52: 743-753, 1975.

8) Norris RM, Whitlick RML, Barratt-Boyes C, et al. Clinical measurement of myocardial infarct size. Modification of a method for the estimation of total creatine phosphokinase release after myocardial infarction. Circulation 51: 614, 1975.

9) Wackers FJT, Shoot JB, Sokole EB, et al. Non-invasive visualization of acute myocardial infarction in man with thallium-201. Br Heart J 37: 741, 1975.

10) Burow RD, Pond M, Schafer AW, Becker L. "Circumferential Profiles": Anew method for computer analysis of thallium-201 myocardial perfusion 


\section{HiRAYAMA et al}

\section{Antero-Septal MI Group}
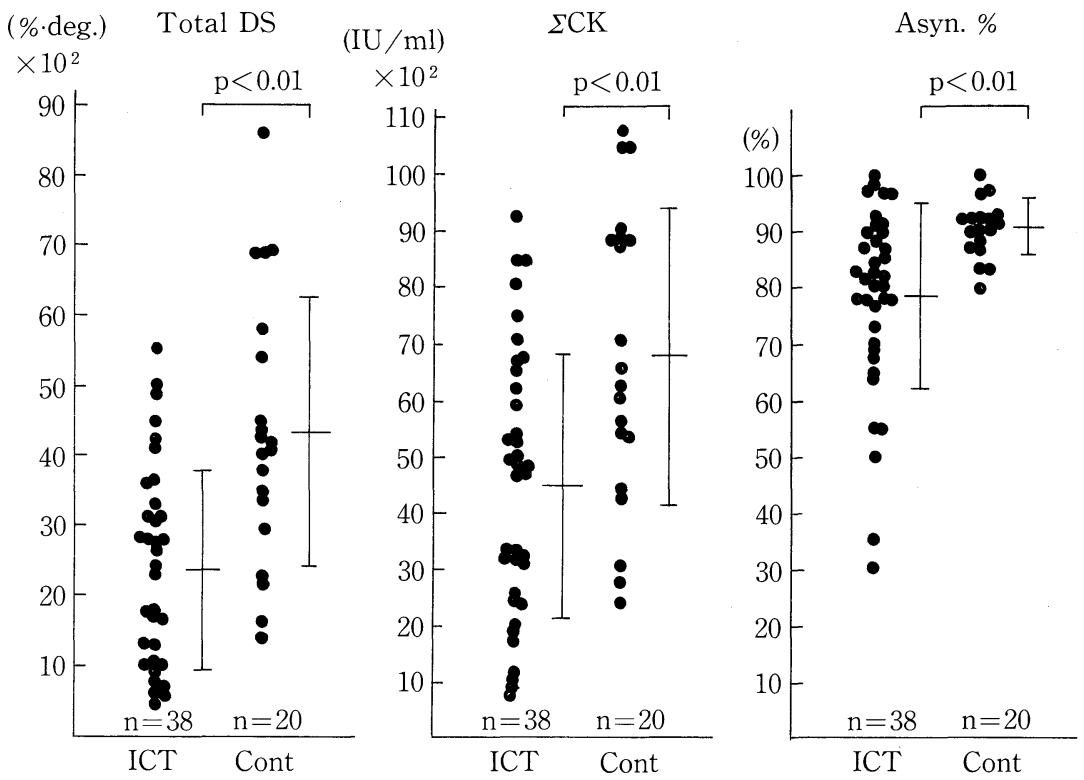

Inferior MI Group
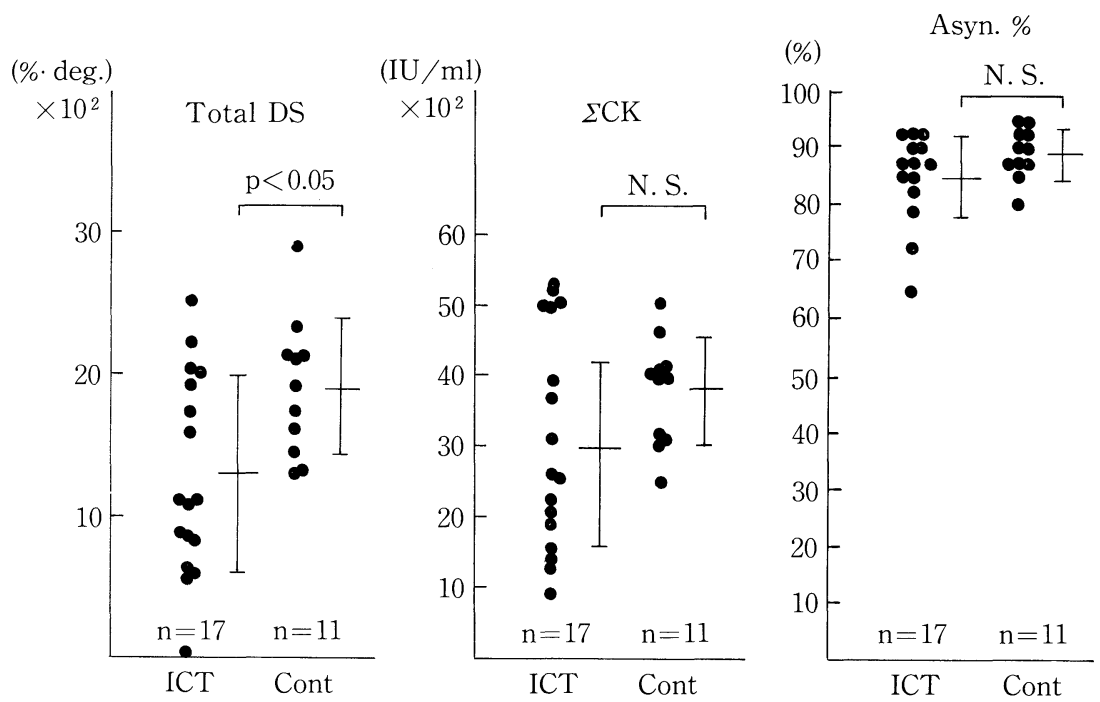

Fig. 4. Comparison of the intracoronary thrombolysis (ICT) and control groups stratified into an anteroseptal MI subgroup and an inferior MI subgroup.

images. J Nucl Med 20: 771, 1979.

11) Sheehan FH, Bolson EL, Dodge HT, et al. Advantages and applications of the center-line method for characterizing regional ventricular function. Circulation 74: 293, 1986.

12) AHA Committee Report. A reporting system of patients evaluated for coronary artery disease. Circulation 54: 5, 1975.

13) Uematsu $\mathrm{K}$, Hirayama $\mathrm{H}$. Comparison of the defect size estimated by the enzymologic method and that estimated by $\mathrm{Tl}-201$ myocardial scintigraphy. Jpn Circ J 48 (Suppl II): 265, 1984 (in Japanese).

14) Mahmarian JJ, Pratt CM, Borges-Neto S, Cashion WR, Roberts R, Verani MS. Quantification of infarct size by Tl-201 single-photon emission computed tomography during acute myocardial infarction in humans: comparison with enzymatic estimates. Circulation 78: 831, 1988.

15) Ingels NB Jr, Mead CW, Daughters GT II, Stinson EB, Alderman EL. A new method for assessment of left ventricular wall motion. Comput Cardiol 57, 1978.

16) Stewart DK, Dodge HT, Frimer M. Quantitative analysis of regional myocardial performance in coronary artery disease. Cardiovascular Image Processing 72: 217, 1975.

17) Jeppson GM, Clayton PD, Blair TJ, Liddle HV, Jensen RL, Klausner SC. Change in left ventricular wall motion after coronary artery bypass surgery: Signal or noise? Circulation 64: 945, 1981. 


\section{Efficacy of Intracoronary Thrombolysis}

18) Gelberg HJ, Brundage BH, Glatz S, Parmley WW. Quantitative left ventricular wall motion analysis: A comparison of area, chord and radial methods. Circulation 59: 991, 1979.

19) Rahimtoola SH. A perspective on the three large multicenter randomized clinical trials of coronary bypass surgery for chronic stable angina. Circulation 72 (Suppl. V): 123, 1985.

20) Braunwald E, Rutherford JD. Reversible ischemic left ventricular dysfunction: Evidence for the hibernating myocardium. J Am Coll Cardiol 8: 1467, 1986.

21) Kolibash AJ, Lewis RP. Improvement of myocardial perfusion and left ventricular function after coronary artery bypass grafting in patients with unstable angina. Circulation 59: 66, 1979.

22) Bateman TM, Berman DS. Transient pathologic $Q$ waves during acute ischemic events: An electrocardiographic correlate of stunned but viable myocardium. Am Heart J 106: 1421, 1983.

23) Hirayama $\mathrm{H}$. A case in which cardiac function deteriorated as the region that was supplied by the occluded coronary artery and showed akinesis after PTCR became dyskinetic 8 months later due to reocclusion of the same vessel. Jpn Circ J 49 (Suppl I): 98, 1984 (in Japanese).

24) Schofer J, Mathy DG. Lack of correlation after reperfusion between ventricular function and infarct size estimated by thallium single-photon emission computed tomography. Int J Cardiac Imaging 3: 203, 1989.

25) Ando A, Hirayama $H$. Changes in cardiac function and viability of the infarcted myocardium 1 or more years after the onset of myocardial infarction. Jpn Circ J 54 (Suppl I): 68, 1990 (in Japanese). 